\title{
WISH cages: constructing multiple habitats for captive chimpanzees
}

\author{
Tetsuro Matsuzawa ${ }^{1,2,3}$
}

Published online: 6 March 2020

(C) Japan Monkey Centre and Springer Japan KK, part of Springer Nature 2020

\section{Introduction}

This article aims to introduce environmental enrichment for captive chimpanzees in Japan in a historical context. It describes the most recent endeavor of constructing huge cages and connecting multiple habitats. Kyoto University has two facilities for chimpanzees. One is at the Primate Research Institute (PRI) in Inuyama, and the other, run by the Wildlife Research Center (WRC), is the Kumamoto Sanctuary (KS) in Kumamoto. There are a group of 12 chimpanzees in PRI and 53 chimpanzees and 6 bonobos in KS. There are 307 chimpanzees in Japan at present. Thus 21\% of all chimpanzees in Japan are kept under the umbrella of Kyoto University.

The Ai Project, a series of studies into the mind of chimpanzees, is based at Kyoto University PRI and started on November 10, 1977. The 42-year-old project has produced many important scientific findings (Matsuzawa et al. 2006) and was the driving force behind promoting environmental enrichment for captive chimpanzees. See the website for the on-going project: https://langint.pri.kyoto-u.ac.jp/ai/

Our original enrichment efforts in the 1990s consisted of providing an open-air outdoor compound that had 15-m-high climbing frames, living trees, and a small stream. The most recent endeavor involves what we call "WISH" cages (see explanation below), which have been constructed in PRI and KS. The idea is to provide chimpanzees with two huge cages adjacent to their existing outdoor compound. These large structures are also interconnected by a corridor. The chimpanzees can freely use these multiple habitats by moving between them at will. This setting simulates the fission-fusion dynamics of chimpanzee groups in the wild. The huge cages are also equipped with a computer-controlled

Tetsuro Matsuzawa

matsuzawa.tetsuro.8w@kyoto-u.ac.jp

1 Kyoto University Institute for Advanced Study, Kyoto, Japan

2 Primate Research Institute, Kyoto University, Inuyama, Japan

3 Japan Monkey Centre, Inuyama, Japan touch panel system for cognitive tests, which serve as an additional enrichment device giving extra feeding opportunities.

\section{CCCC: conservation and welfare of chimpanzees}

Jane Goodall published a landmark book Chimpanzees of Gombe: Patterns of Behavior in 1986 (Goodall 1986). To celebrate the publication, chimpanzee researchers came together in Chicago in November 1986. The meeting resulted in the publication of a symposium-based book Understanding Chimpanzees (Heltne and Marquardt 1989).

I was invited to give a talk at that meeting probably because I had just published an article on Chimpanzee Ai in Nature (Matsuzawa 1985). Chimpanzee Ai is the first

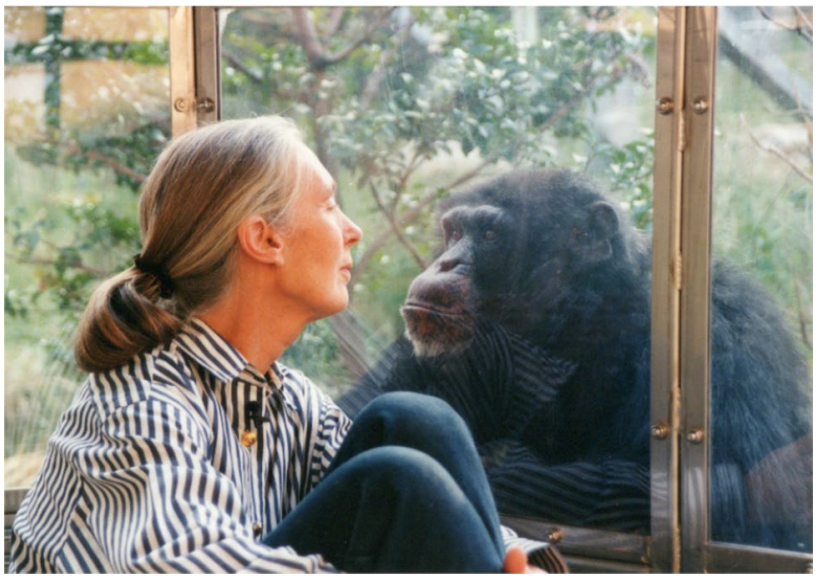

Fig. 1 Jane Goodall and chimpanzee Akira looking at each other. Jane is sitting inside the "gazebo" that is connected to the main building through an underground tunnel. The gazebo reversed the traditional idea of experiments: a human experimenter was kept inside of the room and the chimpanzees were free on the outside. Freedom is at the center of environmental enrichment for chimpanzees. Photo was taken in 1995 by Tetsuro Matsuzawa 
chimpanzee who mastered the use of Arabic numerals to represent the concept of numbers. She succeeded in assigning labels of object names, colors, and numbers to various sample items, e.g., "5 red pencils" or "4 yellow keys". During my talk Jane was sitting in the front row. After my talk she raised a question: "Chimpanzee $\mathrm{Ai}$, what is she doing the rest of the day?" I almost immediately recognized the point of her question. It was clearly the issue of animal welfare. She was interested in knowing about the everyday lives of the chimpanzees who were participating in laboratory-based cognitive studies. Even though the study itself might be of scientific interest, Jane was concerned about the quality of life of the participants who lived in a captive environment (Fig. 1).

At the time of the Chicago symposium, I was spending my sabbatical leave of 1 year and 10 months at the University of Pennsylvania. I also took the opportunity to visit Bossou, Guinea, West Africa, to see chimpanzees in the wild. I spent a month there in February-March 1986 thanks to Prof. Yukimaru Sugiyama, who established the research site at Bossou. I had two questions in my mind for possible fieldwork on wild chimpanzees. First, how was the intelligence of captive chimpanzees actually used by their counterparts in their natural habitat? Second, what is the way of life in the forests: what do chimpanzees eat, how do they move, where do they sleep, and how do they interact with each other? I saw wild chimpanzees use a pair of stones to crack open oil-palm nuts in the dense bush of Bossou's secondary forests. At that time, the Bossou chimpanzee group numbered about 20 individuals. They lived in a fission-fusion society in which the whole community was daily split into smaller parties that travelled through the forest together and reunited with the rest of the group later. I witnessed the close relationship between mothers and their offspring as well. I can never forget the very first day when I watched wild chimpanzees, in February 1986. A mother named Jire was moving with her baby, Ja, through the trees above my head. They were free in the forest. I understood that freedom was the most important and valuable aspect of living in the wild (Fig. 2).

Experience of studying wild chimpanzees in the field is important, perhaps even essential for informed thinking about how to improve the captive environment. In parallel with my efforts to study chimpanzee cognition, I studied Japanese monkeys in the wild (Matsuzawa 2018), including field experiments on food aversion learning in their natural habitat (Matsuzawa et al. 1983) and recording the spatial deployment of snow monkeys in the snow (Wada and Matsuzawa 1986).

My answer to Jane Goodall in Chicago was as follows: Ai was living with the other members of the PRI community, in a social group of 11 chimpanzees. She was free to move around the outside enclosure with her friends. When I needed Ai for cognitive testing, I called her name and she

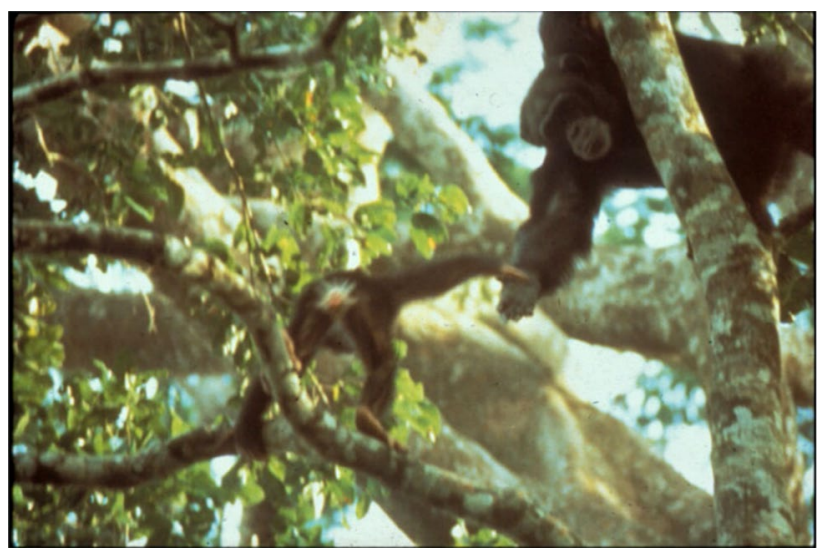

Fig. 2 Wild chimpanzees at Bossou, Guinea, West Africa. A mother named Jire gives a helping hand to her 2.5-year-old daughter named Ja. Photo was taken in February 1986 by Tetsuro Matsuzawa

would decide whether or not to come to the test booth on the basis of her free will. Jane looked at me and smiled: she seemed happy to hear my explanation.

CCCC was proposed by Jane and established during the Chicago meeting. It stands for the Committee for the Care and Conservation of Chimpanzees. Each country was asked to establish a national branch of CCCC. Jane asked Toshisada Nishida (1941-2011) and me to work together to start CCCC-Japan, in which Nishida took the lead on the conservation side and me on the captive care side. Some years later CCCC ceased its activity, but CCCC-Japan survived for several years, providing the basis for promoting care and conservation of chimpanzees in Japan. For example, CCCC-Japan and the Mahale Wildlife Conservation Society produced Pan Africa News, a newsletter for the study of wild chimpanzees, founded by Nishida in 1994. Twenty-five years later the newsletter continues to be published, thanks to former students of Nishida.

\section{PRI outdoor compound with high climbing frames}

After returning to Japan from Africa and sabbatical leave, I set to work to further improve the captive environment of PRI chimpanzees (Matsuzawa 2006). In 1989, Prof. Sugiyama and I decided to take two of our chimpanzee caretakers to Bossou, West Africa, to acquaint them with the real life of wild chimpanzees. Thanks to those caretakers, we started several environmental enrichment projects for the PRI chimpanzees. In 1993 PRI received funding for the creation of a new section of Language and Intelligence (L\&I) to study the chimpanzee mind. As the first professor of L\&I, I oversaw the construction of a new chimpanzee facility. The construction work ended in March 1995, with an outdoor 
compound containing 8-m-high climbing frames, specially planted trees, and a stream (Fig. 3). The height of the tower was increased to $15 \mathrm{~m}$ in 1998. Conditions in the outdoor compound continued to improve year on year (Fig. 4).

Before us, no-one had dared to plant trees in captive chimpanzee environments - it was assumed that they would not last long. However, our trial was successful. Of the various kinds of trees that we planted in the outdoor compound, all the coniferous trees survived. Chimpanzees loved to be able to eat leaves and flowers whenever they wanted (Fig. 5). One of the highlights of our initial attempt at environmental enrichment was a "gazebo". The gazebo is a small glasswalled room constructed in the outdoor compound. Humans can access the gazebo through an underground tunnel connecting it to the main building. Thus, the traditional idea of the laboratory was now reversed: humans were kept inside of the room while chimpanzees were free on the outside. From inside the gazebo, experimenters could set up devices such as orange juice dispensers (Tonooka et al. 1997). Chimpanzees collected twigs and modified them by removing leaves to create tools for drinking. This simulated use of leaf-tools for drinking water by wild chimpanzees (Tonooka 2001; Sousa et al. 2009). Drilling a tiny hole in the acrylic glass also provided the opportunity for honey-fishing (Celli et al. 2004), a simulation of ant-fishing by chimpanzees in Mahale, Tanzania (Nishida 1973; Nishie 2011).

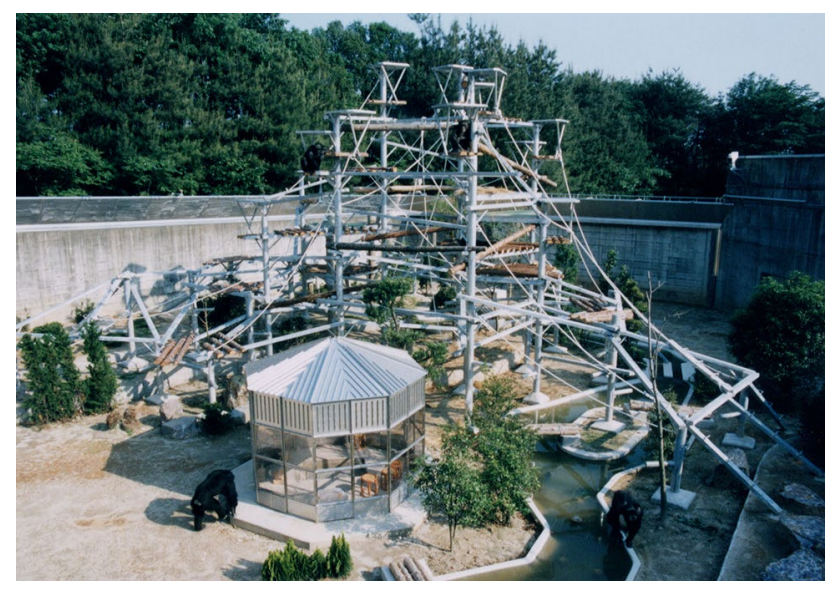

Fig. 3 The outdoor compound at PRI, built in March 1995. Chimpanzee Akira can be seen walking towards the gazebo. The original climbing frames were only $8 \mathrm{~m}$ in height. The trees and shrubs had just been planted when this photo was taken. An artificial stream is visible on the right, through which water was pumped and then recycled. The original idea of the PRI compound consists of high climbing frames, planted trees, a stream, and a gazebo for humanchimpanzee interaction. Photo was taken in March 1995 by Tetsuro Matsuzawa

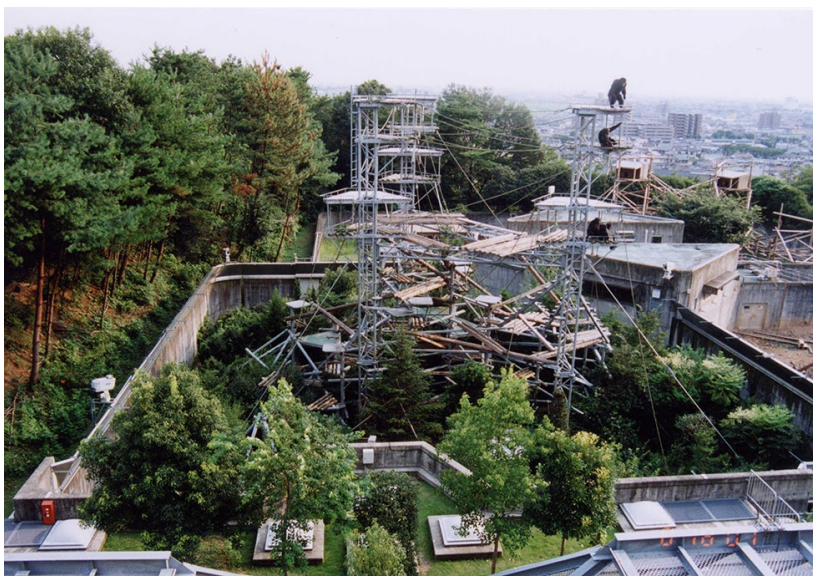

Fig. 4 The PRI outdoor compound continues to change year by year thanks to the growing trees. The height of climbing frames was doubled to $15 \mathrm{~m}$ in 1998. Chimpanzees love to stay on the upper levels: they spend about $80 \%$ of each day in the frames and about $20 \%$ on the ground. Photo was taken by Tetsuro Matsuzawa

\section{SAGA: stop invasive research of chimpanzees}

It was November 20, 1998 when we launched a new endeavor called SAGA (Matsuzawa 2016). SAGA, which

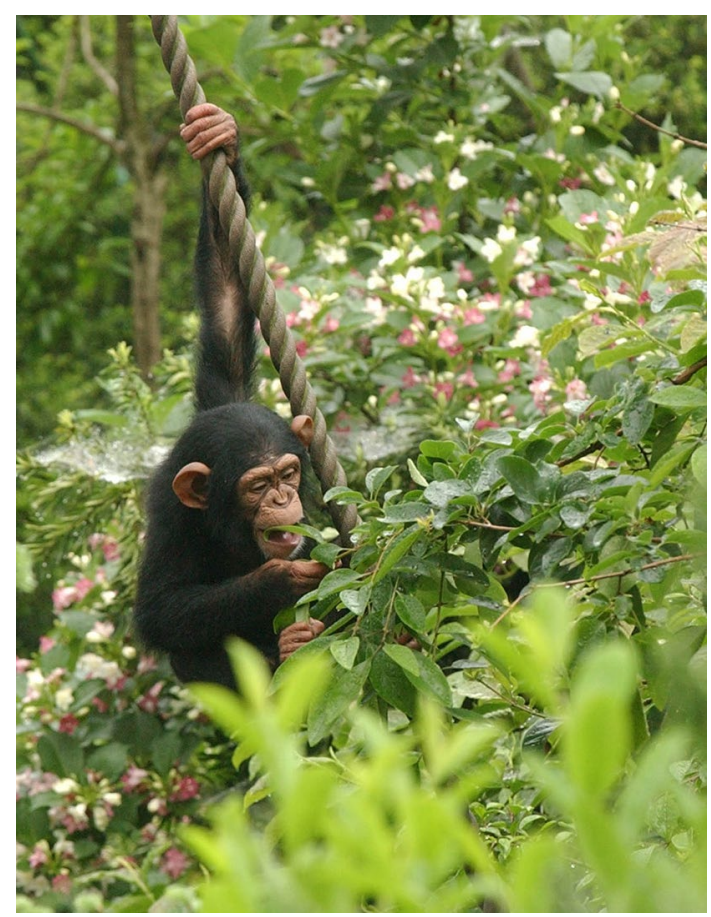

Fig. 5 A young chimpanzee named Cleo is feeding on leaves in the outdoor compound. Thanks to the trees, shrubs, and grasses in the compound, the chimpanzees have the freedom to eat whenever they want, even if the plants are not as tasty as the fruits given by the caretakers. Photo was taken in 2001 by Akihiro Hirata 
stands for Support for African/Asian Great Apes, is a spiritual descendant of CCCC. In collaboration with Juichi Yamagiwa and other colleagues, I started a consortium to promote the conservation and welfare of the great apes. We invited three foreign scholars to the first meeting of SAGA: Jane Goodall for wild chimpanzees, Jan van Hooff for captive chimpanzees, and Alexander Harcourt for wild gorillas. We set up three agendas for SAGA: (1) promoting great ape conservation in their natural habitats, (2) environmental enrichment for improved welfare, (3) prohibiting invasive studies on captive chimpanzees.

Twenty-two years ago, in 1998, 136 chimpanzees were kept by three pharmaceutical companies in Japan. Japan ratified CITES in 1980. It was legal to import wild chimpanzees from Africa in the 1970s, and in that decade more than 150 chimpanzees were imported to Japan, many of them destined for biomedical laboratories in medical schools. Those chimpanzees and their descendants were used mainly for the study of hepatitis $\mathrm{C}$ (it was called hepatitis non-A-non$\mathrm{B}$ then because the $\mathrm{C}$ virus had not yet been discovered). Young, healthy chimpanzees were infected with hepatitis $\mathrm{C}$, and then researchers tried to find a way to cure them. The chimpanzees were kept in small cages $24 \mathrm{~h}$ a day, 7 days a week, because frequent blood sampling was essential for the biomedical studies (Fig. 6). One of the pharmaceutical companies was planning to work on gene therapy for hepatitis $\mathrm{C}$. I decided to take action to stop invasive studies, and that is how SAGA was founded.

SAGA held an annual assembly of people who cared for great apes. Not only scientists but also zoo keepers, veterinarians, administrative officers, media people, and members of the general public who shared a concern for great apes joined the SAGA movement. SAGA facilitated communication between fieldworkers and laboratory researchers, and between scientists and veterinarians and caretakers from the zoo community. CCCC was limited to chimpanzees, whereas SAGA expanded to include all the great apes. In a broader context, social awareness about great apes as endangered species clearly increased.

Thanks to the collective efforts by everyone involved, Japanese pharmaceutical companies unanimously decided to stop invasive studies. All invasive biomedical research on chimpanzees in Japan ceased completely in 2006.

\section{GAIN: a database of great apes}

To stop invasive research on chimpanzees, SAGA started creating a database of all the great apes in Japan. The database together with the associated information network was named GAIN (Matsuzawa 2016). GAIN stands for Great Ape Information Network, and it was launched in April 2002. This program is financially supported by MEXT, a

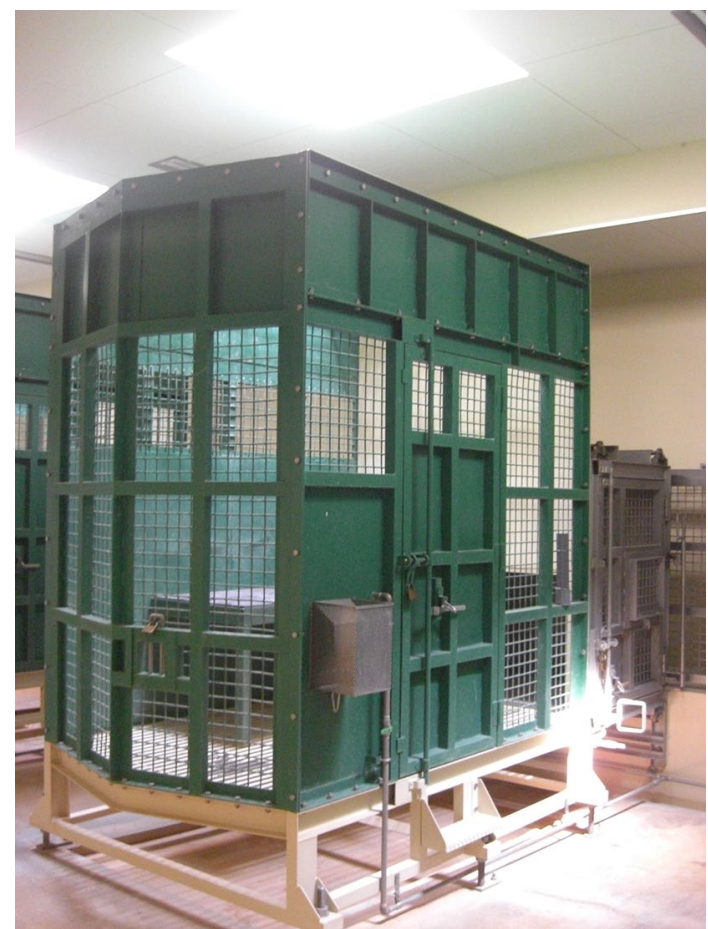

Fig. 6 A cage used in the past in hepatitis $\mathrm{C}$ virus research. Chimpanzees were kept isolated in such single cages to enable routine blood sampling. Photo was taken by Naruki Morimura of Kumamoto Sanctuary

ministry of the Japanese government. The trio of Yasuhiro Yoshikawa (Veterinary medicine, University of Tokyo), Toshikazu Hasegawa (Evolutionary psychology, University of Tokyo), and me (Comparative Cognitive Science, Kyoto University) was in charge of getting funding.

The logic behind GAIN was as follows. Because of CITES, the legal trade in great apes had stopped. This meant that the great apes that were already in Japan were an important bioresource. Of about 90 zoos in Japan, about 50 had chimpanzees. Thanks to SAGA, there was already a close relationship between people from the zoo community and the researchers, and we collaborated to create the database of individuals and facilities (Fig. 7).

The principal aim of GAIN was the creation of the database, but it also targeted the facilitation of postmortem utilization of chimpanzees. There remain potential needs for captive chimpanzees not only in biomedical research but also in other domains such as brain science, life sciences, and genome science. Some researchers simply need brain or other tissue samples, blood, bones, parts of organs, and so forth. These needs can often be satisfied just as well by fresh cadavers as by living chimpanzees. This approach may help to reinforce the restriction on invasive studies and help to maintain healthy captive chimpanzees as an important bioresource. For appropriate postmortem utilization, information 


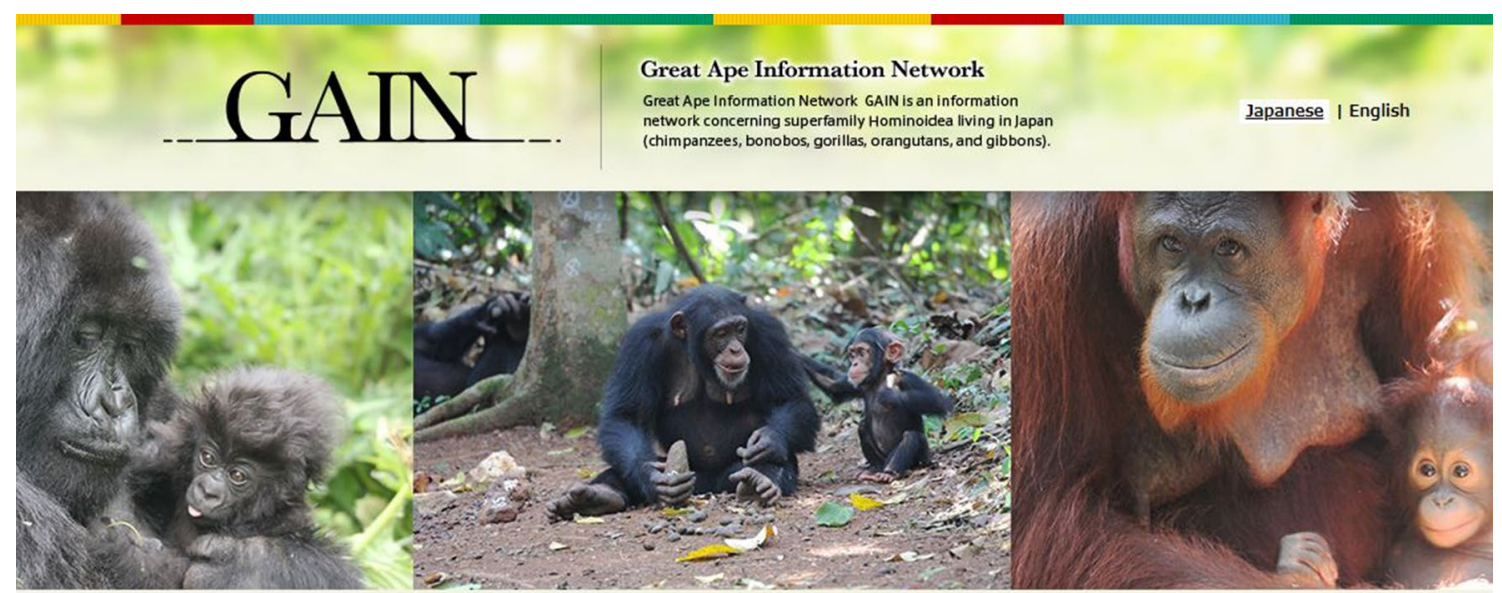

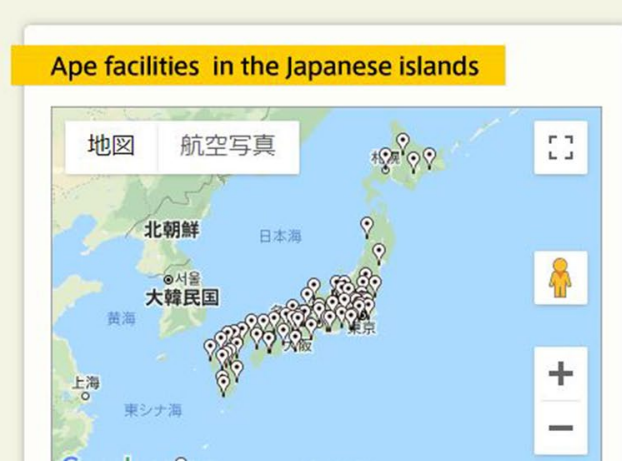

Fig. 7 The front page of the GAIN website. GAIN, Great Ape Information Network, is a database of all apes living in Japan. Visitors to the site can, at a glance, find out where each ape is in Japan. The database also includes individuals who have already passed away.

on all chimpanzees in Japan is essential: we need to identify each individual and track births, deaths, and relocations as quickly as possible. GAIN is an online database that allows us to check who is in which zoo at any given moment. Now, in February 2020, there are 307 chimpanzees across 48 facilities in Japan.

GAIN expanded to include not only chimpanzees and bonobos but also gorillas, orangutans, and even gibbons. As of February 2020, there are 6 bonobos in one facility, 20 gorillas across 6 facilities, 45 orangutans across 19 facilities, and 172 gibbons across 43 facilities. All individuals have a GAIN number and genome information, and their pedigree is recorded. The studbooks of species kept in zoos are not open to the public. However, GAIN aimed to make such information open to the public anywhere in the world with internet access: anyone can consult the information in Japanese and in English.

The GAIN database provides a unique opportunity for researchers to analyze the personal data of chimpanzees who are registered in Japan. The first chimpanzee in Japan arrived in 1921. Since then about 1000 chimpanzees have been kept in Japan, of which around $70 \%$ are deceased. We calculated

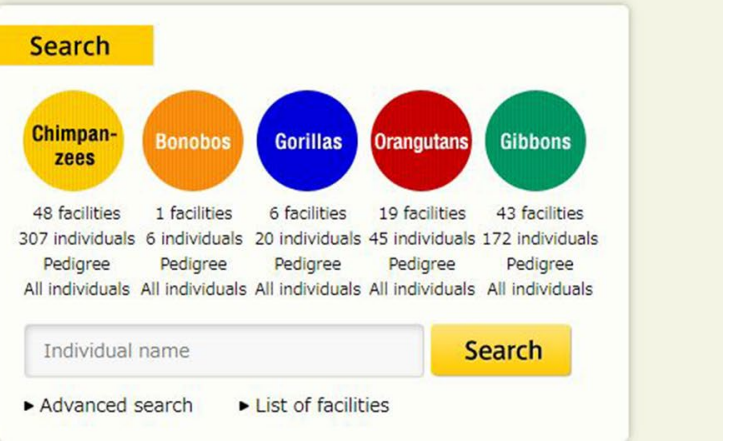

This provides information on the pedigree of each individual. Genetic information and personality profiles are also open to the public both in English and in Japanese

life expectancy based on 821 of those captive chimpanzees for whom we have precise records of age at death (Havercamp et al. 2019). The average was 28.3 years.

The average life expectancy at birth is the simple average of all 821 individuals. However, we know that about 20\% of those individuals died soon after birth because of illness, accidents, or other causes. Omitting such cases, suppose that a baby chimpanzee survives for one full year. Then the average life expectancy of those chimpanzees increases to 40.4 years; they survive for 40 years on average. The longest record among the 1000 individuals in Japan belongs to Jonny (GAIN number 0003V) who passed away on January 15,2019 at the age of 68 years. Thus, GAIN provides a statistical starting point for captive care and management.

One chimpanzee housed in KS was identified to have Down syndrome, only the second known chimpanzee case in the world (Hirata et al. 2017). The first trio genome, i.e., whole-genome comparison among father-mother-son, was reported in Akira-Ai-Ayumu chimpanzees in PRI (Tatsumoto et al. 2017). The aging community of captive chimpanzees in Japan will continue to provide us with unique 
opportunities to understand human aging from a comparative perspective.

Please visit GAIN for further information: https://shige n.nig.ac.jp/gain/.

\section{WRC and Kumamoto Sanctuary}

After working to stop invasive studies on chimpanzees, we were faced with another problem. There were surplus ex-biomedical chimpanzees who no longer brought any financial benefits to the pharmaceutical companies. It was difficult to ask those private companies to keep their chimpanzees as a goodwill gesture; instead, they asked us, SAGA, for a solution. We decided to create the Wildlife Research Center (WRC) of Kyoto University. The center aimed to focus especially on endangered middle- to large-size animals such as chimpanzees. I was the Director of the Primate Research Institute (PRI) of Kyoto University from 2006 to 2012. I asked my colleagues to agree with the plan to assign human and financial resources to create a new center for wildlife. PRI helped in founding the new sister center, WRC, in 2008. WRC had six tenured professors, all focused on conservation and welfare of wild animals.

The largest pharmaceutical company donated the chimpanzees, the land, and the buildings to WRC. As a result, WRC created Kumamoto Sanctuary (KS) in August 2011 (Morimura et al. 2010). KS is a section of WRC which runs a retirement sanctuary for ex-biomedical chimpanzees. The chimpanzees that belonged to the other two pharmaceutical companies also joined KS.

May 15, 2012 is a memorable day for the chimpanzees in Japan. Thanks to the effort of Satoshi Hirata and his colleagues, the last three chimpanzees kept in small individual cages were freed and brought to KS. Those chimpanzees had never seen the sun, trees, or other natural things. They had never felt a breeze or stepped on bare soil in more than 30 years (Hirata et al. 2012). All the ex-biomedical chimpanzees had lived in single cages, but now they enjoy a social life and are at no risk of ever being used for invasive research (Fig. 8).

Please visit WRC: https://www.wrc.kyoto-u.ac.jp/en/ index.html.

Please visit KS: https://www.wrc.kyoto-u.ac.jp/kumasan/ indexE.html.

\section{WISH: cages connecting multiple habitats}

Freedom from fear was the crux of the matter: the ex-biomedical chimpanzees were freed. What was the next step? We reflected on our experiences in the wild to make further efforts to improve the captive situation. In my opinion, there

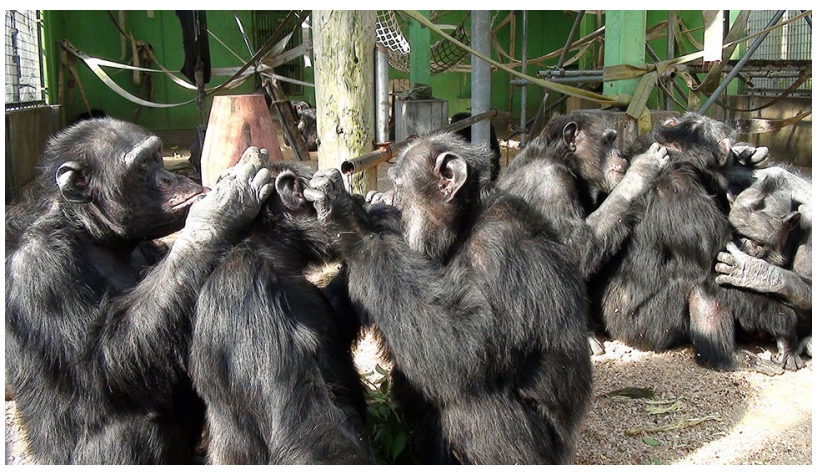

Fig. 8 All-male group of Kumamoto Sanctuary. Previously singlehoused chimpanzees were introduced one by one to create a stable bachelor group. Photo was provided by Kumamoto Sanctuary

are two major differences between the wild and captivity in terms of animal welfare. The first is the freedom to eat. Chimpanzees in the wild can forage and eat whenever they want. On the contrary, chimpanzees in captive facilities cannot eat freely; their eating schedule depends on the caretakers who bring them food. The second major difference is the freedom to move. Chimpanzees in the wild can go whenever and wherever they want. On the contrary, chimpanzees in captive facilities cannot; they are confined to a limited area and are permanently in the company of the other members of the community.

On the basis of the need for freedom of movement, I imagined a captive environment of multiple connecting habitats. The sister institutes, PRI and WRC-KS, sought to earn a good international reputation and wide attention (de Bezerra 2018; Langlitz 2017). The cognitive studies in Kyoto University were crystalized into a new endeavor called WISH, Web for the Integrated Study of Human mind. In 2010, the Japanese government (via the Ministry of Education, Culture, Sports, Science and Technology) chose WISH as one of 14 big projects in the country. Within the framework of WISH, we wanted to turn a dream into reality. My colleagues and I created a plan to construct huge cages that were interconnected, to actualize multiple habitats for our captive chimpanzees.

We constructed two WISH cages in PRI, Inuyama, and another two in WRC-KS, Kumamoto. They are called cages, but they are not small; in fact they are huge, 10-20 m long and up to $16 \mathrm{~m}$ in height. The height is equivalent to the 6th floor of a building. The construction work took place over 4 years from 2011 to 2014, and it cost a little more than 10 million US dollars in total.

In PRI, Inuyama, we built WISH cage \#1 (silver cage) and WISH cage \#2 (green cage). Both cages faced onto the outdoor compound with trees (Figs. 9, 10). The two cages were also interconnected by a corridor at the level equivalent to the 4th floor. Members of the group of 12 chimpanzees 


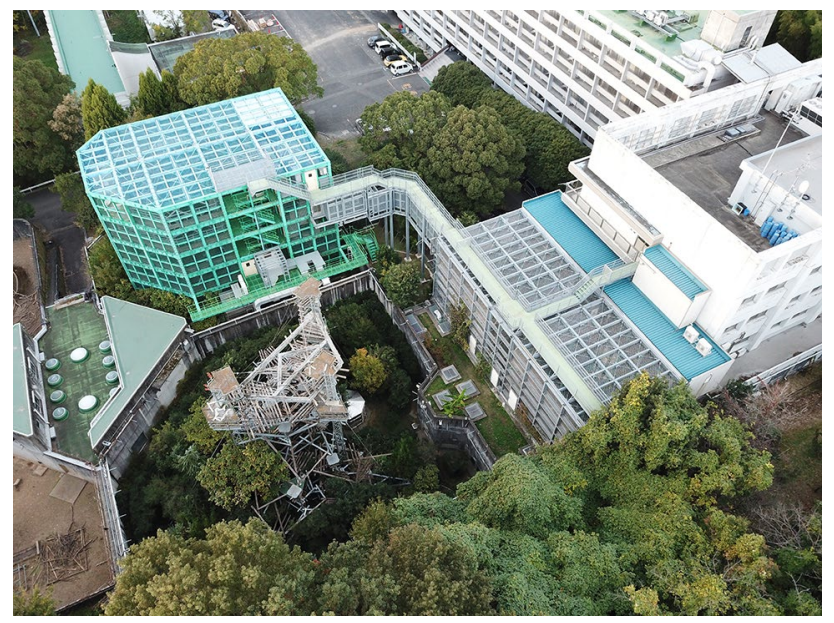

Fig. 9 View of the three habitats in PRI in Inuyama. There are two huge WISH cages facing the outdoor compound. The silver cage located to the right is called WISH cage \#1 PRI. The green cage located to the left is called WISH cage \#2 PRI. The two cages are interconnected by a corridor located at the top floors of the two cages. The chimpanzees can freely move from one place to another in fission-fusion parties. Photo was taken by drone in November 2019 by Tetsuro Matsuzawa

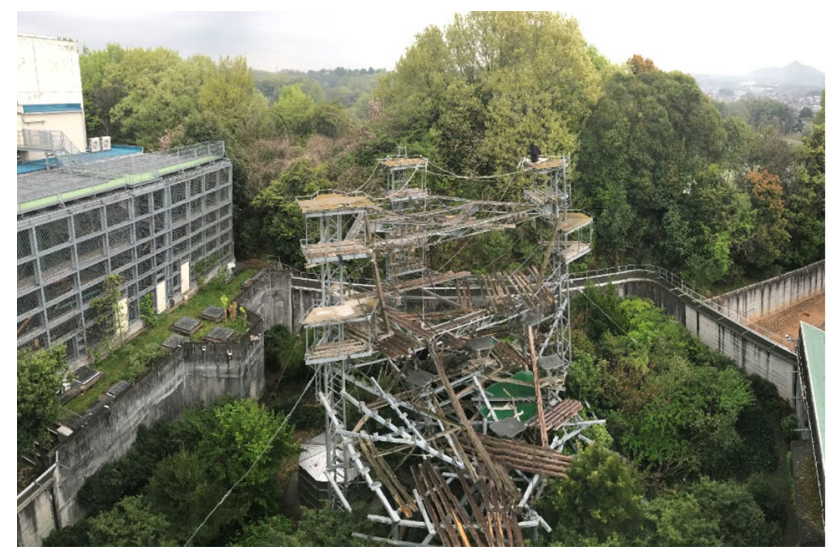

Fig. 10 Climbing frames, called the "triple tower", in the outdoor compound in PRI. A chimpanzee is visible sitting on the top of the right tower that is $15 \mathrm{~m}$ high. WISH cage \#1 PRI (silver) is located to the left of the outdoor compound. The two habitats are connected by doors in the walls. The photographer is located on the viewing terrace on top of WISH cage \#2 PRI (green). Photo was taken in November 2019 by Tetsuro Matsuzawa

were free to move from one place to another among the three habitats: cage \#1, cage \#2, and the adjacent outdoor compound. The multiple habitats allow the chimpanzees to split into small groups, with one group here, another group there, while a third group might be in the outdoor compound. The multiple habitat setting actualizes the fission-fusion society of wild chimpanzees.
The freedom to eat was operationalized through the Skylab in WISH-Inuyama cage \#1. The Sky-lab is a laboratory space inside WISH cage \#1. From the viewpoint of a chimpanzee inside the cage, the Sky-lab is located at the height of the 4th floor of the building. Chimpanzees can access one of eight booths attached to the laboratory, each equipped with a computer-connected touch panel and automated food dispenser. To know which chimpanzee is in front of the touch panel, Ikuma Adachi and his colleagues invented a fully automated chimpanzee face recognition system.

The idea of Sky-lab stems from the principle of freedom to eat. The doors to the eight booths can always be open. The video cameras connected to the computer identify who is in front of the computer. We invented a new food dispenser that can hold 300 pieces of food, three times more than the usual dispenser. This means that chimpanzees can access food whenever they want, although they have to solve a cognitive test to get the reward. Chimpanzees are free to come to the booth and work for small treats, and free to leave whenever they want.

The Sky-lab in WISH-Inuyama cage \#1 corresponds to what we named the interaction booths in WISH-Inuyama cage \#2 (Fig. 11). The interaction booths are located at the equivalent of the 6th floor of the building. Wild chimpanzees climb up to such heights in the tree canopy to feed on fruits there. A chimpanzee on the ground in cage \#2 wanting to work for food has to use the climbing frames to reach the top and enter the booth, which is equipped with three touch screen panels. Interactions among groups of chimpanzees

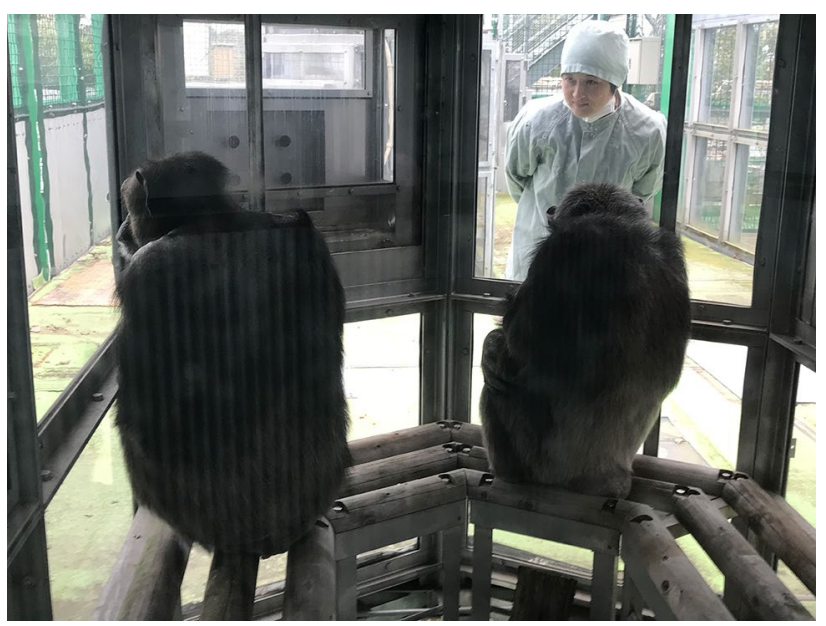

Fig. 11 "Interaction booth" located inside of WISH cage \#2 PRI (green one). It is located at the top floor of the huge cage. The chimpanzees can access the booth from the bottom. It is designed as an image of the tree canopy. The chimpanzees are using climbing frames to reach the interaction booth equipped with three sets of computercontrolled touch panel system. They can interact with each other and face the human observer at a close distance as well through the translucent panels. Photo was taken in 2019 by Tetsuro Matsuzawa 
engaging in cognitive tasks are thus studied. The WISHInuyama cages and the connecting corridor officially started to be used by the 12 chimpanzees on October 8, 2015.

Two WISH cages were also built in WRC-KS, Kumamoto (Fig. 12). Kumamoto Sanctuary is located on the slope of a small mountain near the Ariake sea. Cage \#1 was built at the bottom of the slope and cage \#2 was built higher up the slope (Figs. 13, 14). The two huge cages are interconnected by a 150 -m-long corridor. When the doors of the long corridor are open, the chimpanzees can move from one cage to the other. The chimpanzees are much calmer than we initially expected when they pass through the corridor, which they often do in a line (Fig. 15).

Kumamoto Sanctuary was renovated by building the WISH cages so that we could receive bonobos from North America. The Bonobo Survival Plan offered six bonobos to Japan. They are currently the only bonobos in this country. Therefore, KS provides a rare opportunity for researchers to compare chimpanzees and bonobos at the same facility (Kano et al. 2015; Sato et al. 2019).

\section{Looking back at the past to think about the future}

The history of studying the chimpanzee mind in Japan is also the history of environmental enrichment in this country. The first high climbing frames were built in PRI in 1995. The climbing frames allow three-dimensional space utilization for captive chimpanzees. The idea of high climbing frames has spread to numerous zoos in Japan such as Tama, Sapporo, Asahiyama, Higashiyama-Nagoya, and Kyoto, as well

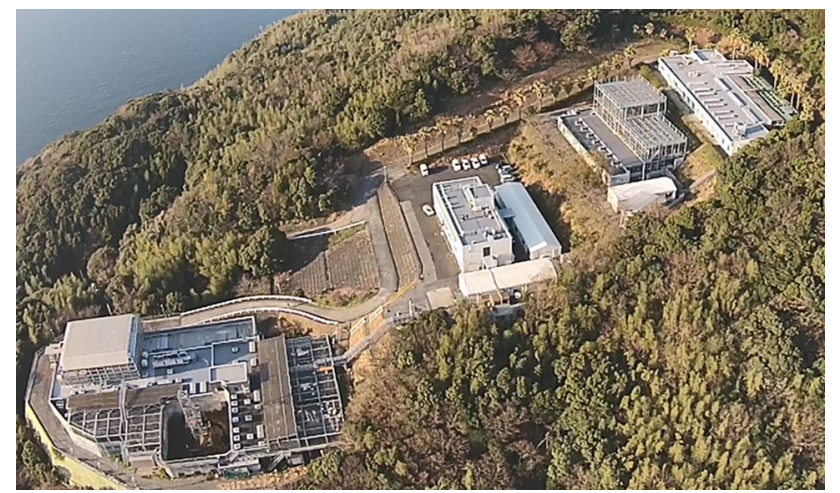

Fig. 12 Overview of Kumamoto Sanctuary (KS) in Kumamoto prefecture that is facing the Ariake sea. WISH cage \#1 in KS is located to the top left corner that is covered by a sort of roof. WISH cage \#2 in $\mathrm{KS}$ is located in the 2 nd building from the right. The two cages are interconnected by a long corridor of $150 \mathrm{~m}$ long. Photo was taken by drone in November 2019 by Naruki Morimura of Kumamoto Sanctuary

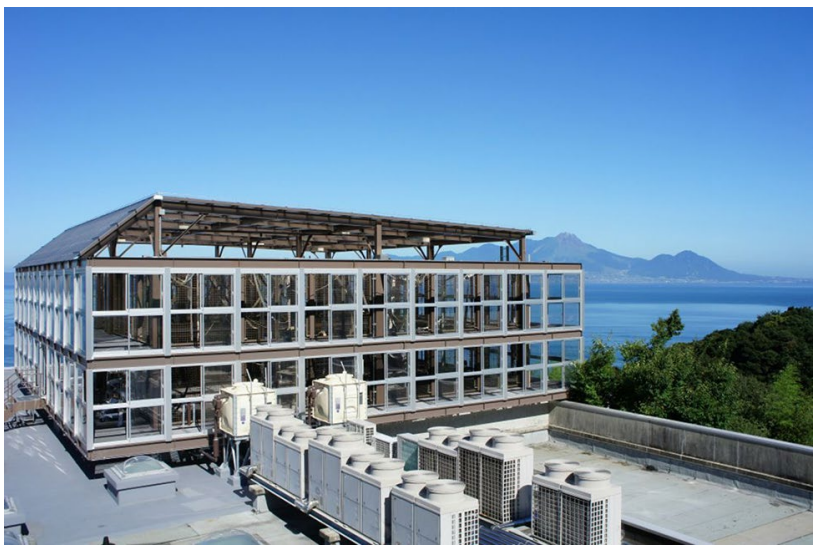

Fig. 13 WISH cage \#1 KS looks like a building. All of the four side walls consisted of translucent panels. The side panels and also the top panels can be removed to allow a natural breeze to enter the huge cage. Photo was provided by Kumamoto Sanctuary

as zoos in other countries, e.g., Edinburgh in the UK and Indianapolis in the USA.

The WISH cages are the result of the desire to further improve captive conditions through environmental enrichment. The essential aspect of the WISH cages is the connections between the multiple habitats to allow the fission-fusion movement of chimpanzees. The WISH cages are also unique in terms of providing cognitive testing spaces. The human laboratory space contains computer-controlled touch panel systems as well as the fully automated video camera system to identify which chimpanzee has chosen to use a given touch panel.

Previously, cognitive testing was conducted by isolating one or a pair of chimpanzees in an experimental booth located away from their daily living space. This conventional

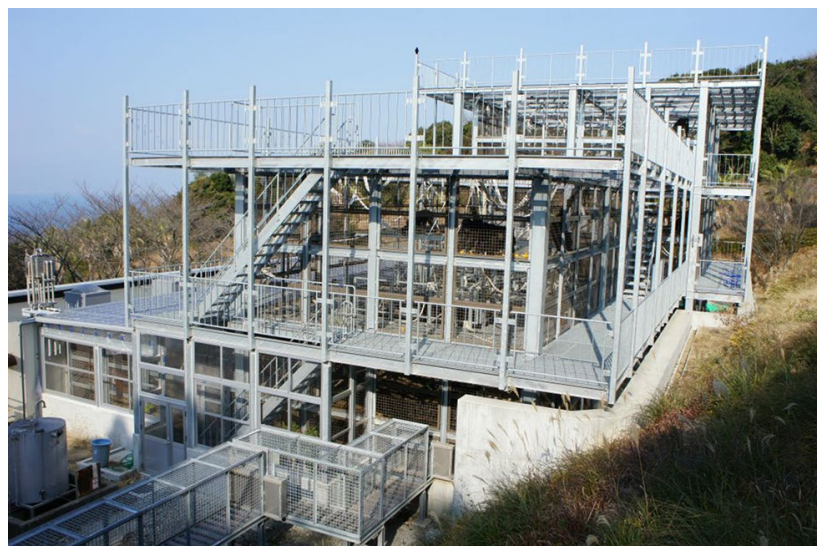

Fig. 14 WISH cage \#2 KS really looks like a cage of three stories. These outside areas are the floors for the human observers. They can observe the chimpanzees from $360^{\circ}$. Photo was provided by Kumamoto Sanctuary 


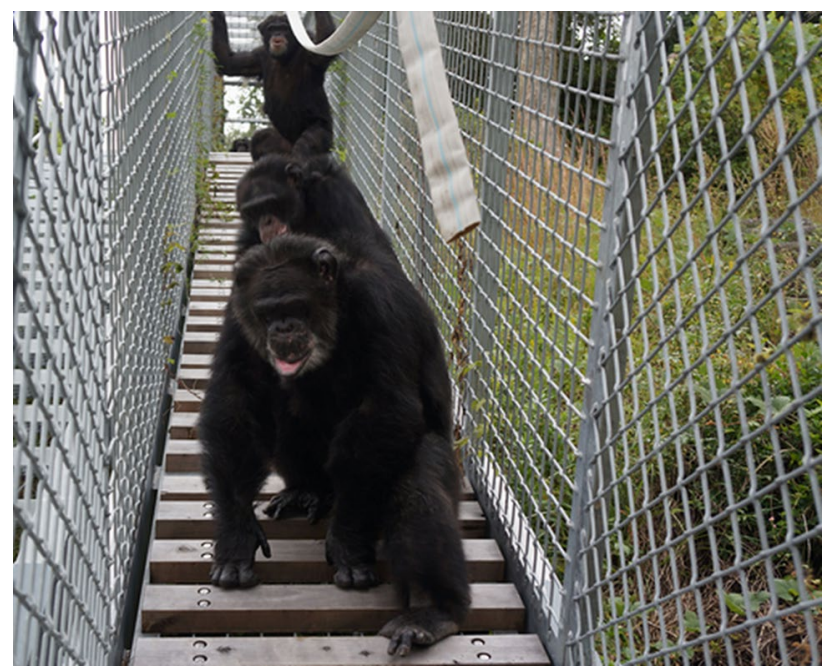

Fig. 15 The chimpanzees in KS calmly move down in the 150 -m-long corridor connecting the remote cages. Photo was provided by Kumamoto Sanctuary

practice was challenged by the WISH cages, which allow the chimpanzees to access food at any time of day or night. By paying attention to the two kinds of freedom, eating and moving, the WISH cages provide us with a glimpse of future approaches to cognitive studies in captivity: social cognition in an enriched environment. In short, efforts to enrich the captive environment can and should go hand-in-hand with sophisticated cognitive studies. In future I hope to see many other facilities following the concept of the WISH cages, connecting multiple habitats and providing more freedoms for captive chimpanzees.

Acknowledgements I looked back to where the idea of WISH cages came from, how it developed, and its aims. Thanks are due to the following persons and organizations. The Ministry of Education, Culture, Sports, Science and Technology, Japan (MEXT) and the Japan Society for the Promotion of Science (JSPS) have provided financial support for studying chimpanzees in the wild and laboratory for decades. I also thank Kyoto University and the Primate Research Institute and the Wild Research Center. The two sister institutions and their personnel work tirelessly to keep the chimpanzees and bonobos in good environments. I thank my four mentors: Prof. Kiyoko Murofushi gave me the opportunity to freely continue running the Ai Project since 1997. Prof. Yukimaru Sugiyama gave me the opportunity to visit Bossou in 1986, resulting in my long-term commitment to wild chimpanzees. David Premack (1925-2015) opened my eyes to the intelligence of chimpanzees. Jane Goodall has been encouraging my motivation to make the lives of captive chimpanzees better. I also thank my junior colleagues at Kyoto University who have been collaborating with me on laboratory studies of the chimpanzee mind: Special thanks are due to Kazuo Fujita, Masaki Tomonaga, Masayuki Tanaka, Satoshi Hirata, Naruki Morimiura, Ikuma Adachi, Misato Hayashi, Yuko Hattori, Yumi Yamanashi, and many younger students. I also owe thanks to foreign collaborators, especially in the wild: Tatyana Humle, Dora Biro, Cláudia Sousa (1975-2014), Laura Martinez, Nicolas Granier, Kathelijne Koops, Kimberley Hockings, Susana Carvalho, and younger colleagues. Without the dedication of those people I could not have reached this place. Finally, I really appreciate the chimpanzees who spent their lives at Kyoto University, and my colleagues in Africa. The study is financially supported by MEXT 16H06283, JSPS core-tocore CCSN, MEXT-JSPS leading graduate program U04 (PWS), and NBRP-Information-GAIN to TM. I also thank Drs. James Anderson and Dora Biro for editing the English text.

\section{References}

Celli ML, Hirata S, Tomonaga M (2004) Socioecological influences on tool use in captive chimpanzees. Int J Prim 25:1267-1281. https ://doi.org/10.1023/B:IJOP.0000043962.60837.16

de Bezerra MDC (2018) Drawing and blurring boundaries between species: an etho-ethnography of human-chimpanzee social relations at the Primate Research Institute of Kyoto University. Ecole doctorale n500, Ph.D. thesis, École Normale Supérieure

Goodall J (1986) The chimpanzees of Gombe: patterns of behavior. Harvard University Press, Cambridge

Havercamp K, Watanuki K, Tomonaga M, Matsuzawa T, Hirata S (2019) Longevity and mortality of captive chimpanzees in Japan from 1921 to 2018. Primates 60:525-535. https://doi.org/10.1007/ s10329-019-00755-8

Heltne PG, Marquardt LA (1989) Understanding chimpanzees. Harvard University Press, Cambridge

Hirata S, Udono T, Tomonaga M, Matsuzawa T (2012) Looking up the sky 30 years later: the biomedical studies of chimpanzees ended in Japan. Kagaku 82:866-867 (In Japanese)

Hirata S, Hirai H, Nogami E, Morimura N, Udono T (2017) Chimpanzee down syndrome: a case study of trisomy 22 in a captive chimpanzee. Primates 58:267-273. https://doi.org/10.1007/s1032 9-017-0597-8

Kano F, Hirata S, Call J (2015) Social attention in the two species of Pan: bonobos make more eye contact than chimpanzees. PLoS One 10(6):e0129684. https://doi.org/10.1371/journal.pone.01296 84

Langlitz N (2017) Synthetic primatology: what humans and chimpanzees do in a Japanese laboratory and the African field. BJHS Themes 2:101-125. https://doi.org/10.1017/bjt.2017.4

Matsuzawa T (1985) Use of numbers by a chimpanzee. Nature 315:57-59

Matsuzawa T (2006) Sociocognitive development in chimpanzees: a synthesis of laboratory work and fieldwork. In: Matsuzawa T, Tomonaga M, Tanaka M (eds) Cognitive development in chimpanzees. Springer, Tokyo, pp 3-33

Matsuzawa T (2016) SAGA and GAIN for great apes. Primates 57:1-2. https://doi.org/10.1007/s10329-015-0504-0

Matsuzawa T (2018) Hot-spring bathing of wild monkeys in ShigaHeights: origin and propagation of a cultural behavior. Primates 59:209-213. https://doi.org/10.1007/s10329-018-0661-z

Matsuzawa T, Hasegawa Y, Gotoh S, Wada K (1983) One-trial longlasting food-aversion learning in wild Japanese monkeys (Macaca fuscata). Behav Neural Biol 39:155-159

Matsuzawa T, Tomonaga M, Tanaka M (2006) Cognitive development in chimpanzees. Springer, Berlin

Morimura N, Idani G, Matsuzawa T (2010) The first chimpanzee sanctuary in Japan: an attempt to care for the "surplus" of biomedical research. Am J Primatol. https://doi.org/10.1002/ajp.20887

Nishida T (1973) The ant-gathering behaviour by the use of tools among wild chimpanzees of the Mahali Mountains. J Hum Evol 2:357-370

Nishie H (2011) Natural history of Camponotus ant-fishing by the M group chimpanzees at the Mahale Mountains National Park, 
Tanzania. Primates 52:329. https://doi.org/10.1007/s1032 9-011-0270-6

Sato Y, Hirata S, Kano F (2019) Spontaneous attention and psychophysiological responses to others' injury in chimpanzees. Anim Cogn 22:807-823. https://doi.org/10.1007/s10071-019-01276-z

Sousa C, Biro D, Matsuzawa T (2009) Leaf-tool use for drinking water by wild chimpanzees (Pan troglodytes): acquisition patterns and handedness. Anim Cogn 12:115-125. https://doi.org/10.1007/ s10071-009-0278-0

Tatsumoto S, Go Y, Fukuta K et al (2017) Direct estimation of de novo mutation rates in a chimpanzee parent-offspring trio by ultra-deep whole genome sequencing. Sci Rep 7:13561. https:// doi.org/10.1038/s41598-017-13919-7

Tonooka R (2001) Leaf-folding behavior for drinking water by wild chimpanzees (Pan troglodytes verus) at Bossou, Guinea. Anim Cogn 4:325-334. https://doi.org/10.1007/s100710100110
Tonooka R, Tomonaga M, Matsuzawa T (1997) Acquisition and transmission of tool making and use for drinking juice in a group of captive chimpanzees (Pan troglodytes). Jpn Psychol Res 39:253265. https://doi.org/10.1111/1468-5884.00058

Wada K, Matsuzawa T (1986) A new approach to evaluating troop deployment in wild Japanese monkeys. Int J Primatol 7:1-14

Publisher's Note Springer Nature remains neutral with regard to jurisdictional claims in published maps and institutional affiliations. 